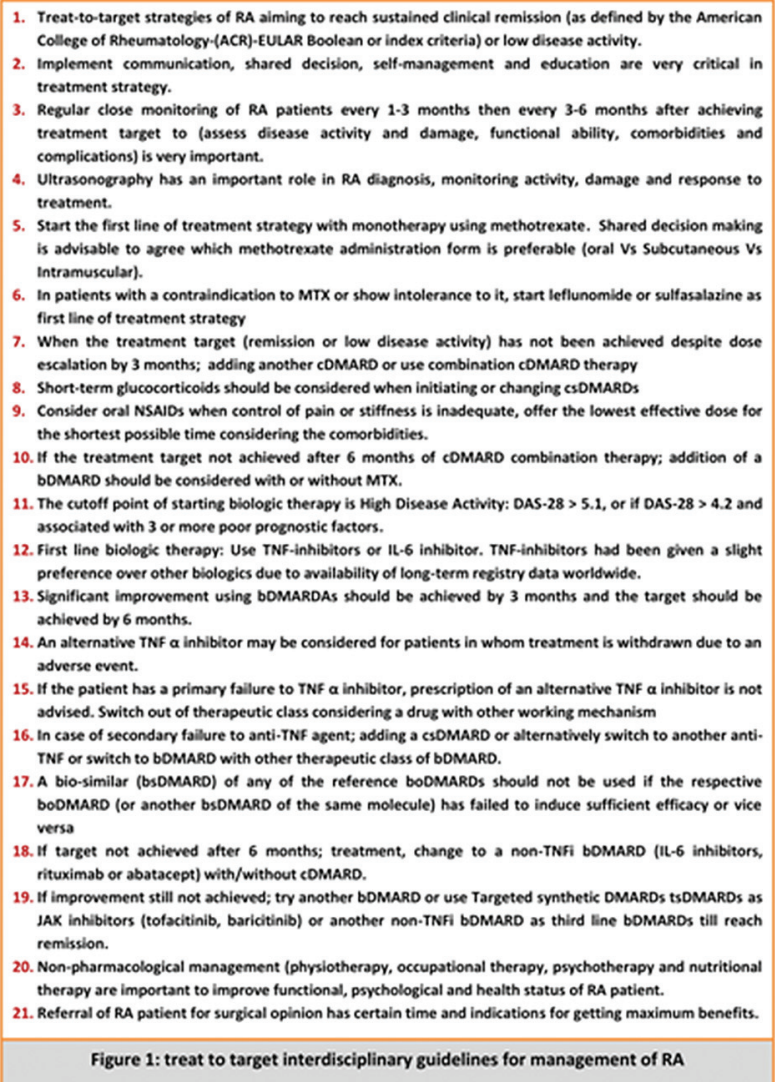

REFERENCES:

[1] Qiang Guo, et al. Rheumatoid arthritis: pathological mechanisms and modern pharmacologic therapies. Bone Res 2018; 6: 15-21.

[2] Smolen JS, et al. EULAR recommendations for the management of rheumatoid arthritis with synthetic and biological disease-modifying antirheumatic drugs: 2016 update. Ann Rheum Dis. 2017; 76(6):960-977.

Disclosure of Interests: None declared

DOI: 10.1136/annrheumdis-2019-eular.2054

\section{FRI0075 SYSTEMATIC ANALYSIS OF INJECTION-SITE PAIN CAUSED BY SUBCUTANEOUS ADMINISTRATION OF THE ADALIMUMAB BIOSIMILAR FKB327 VERSUS ADMINISTRATION OF THE ADALIMUMAB REFERENCE PRODUCT VIA DIFFERENT DELIVERY METHODS}

Rieke Alten ${ }^{1}$, Mark C. Genovese ${ }^{2}$, Malcolm Boyce ${ }^{3}$, Takuma Yonemura ${ }^{4}$, Takahiro Ito ${ }^{5}$, Herbert Kellner ${ }^{6} .{ }^{1}$ University Medicine Berlin, Berlin, Germany; ${ }^{2}$ Stanford University, Division of Immunology and Rheumatology, Palo Alto, CA, United States of America; ${ }^{3}$ Hammersmith Medicines Research, London, United Kingdom; ${ }^{4}$ Souseikai Sumida Hospital, Tokyo, Japan; ${ }^{5}$ Fujifilm Kyowa Kirin Biologics, Tokyo, Japan; ${ }^{6}$ Specialist Practice in Rheumatology and Gastroenterology, Munich, Germany

Background: FKB327 is a proposed biosimilar of the adalimumab reference product (RP). Several studies in both healthy volunteers and patients with active rheumatoid arthritis (RA) were undertaken, the results of which have been reported elsewhere. The formulation excipients of the biosimilar product differ from those of the RP, and different injection-site pain intensity with subcutaneous injection has been reported.

Objectives: The current meta-analysis examines pooled data from these studies in relation to the amount of injection-site pain resulting from using a prefilled syringe (PFS) versus an auto-injector (Al) versus a regular syringe (RS), and the proposed biosimilar, FKB327, versus the RP. Methods: Data from 4 studies, FKB327-001, -002, -003, and -004, were pooled in an effort to compare injection-site pain upon subcutaneous administration of FKB327 versus the RP (citrate-containing formulation of the RP $[40 \mathrm{mg} / 0.8 \mathrm{~mL}])$. Study FKB327-001, in healthy volunteers $(\mathrm{n}=$
180), involved a single subcutaneous dose of either FKB327 or the RP. Study FKB327-004 was a similar study in healthy Japanese volunteers (n $=130$ ). Study FKB327-002 was a randomized (FKB327 with RS or the RP), double-blind, multiple-dose study in patients with active RA. This was followed by Study FKB327-003, in which patients were rerandomized to receive either FKB327 with PFS or the RP in the randomization phase, followed by an open-label extension phase of the study, in which Al was introduced. As patients continued receiving treatment or switched treatments during the course of the FKB327-002 and -003 studies, injection-site pain was assessed at the first dosing occasion of FKB327 or the RP $(n=691)$. Data from all 4 studies were examined by meta-analysis of the visual analog scale (VAS) using a 100-mm horizontal scale for FKB327 versus the RP and for comparison of Al, PFS, and RS.

Results: Data were analyzed from a total of 2007 assessments in 1001 subjects. A linear mixed model of the VAS in $\mathrm{mm}$ for the RP versus FKB327 across all 4 studies showed a 12.6-point lower pain score for FKB327 versus the RP $(95 \%$ confidence interval $[\mathrm{Cl}],-14.3$ to $-10.8 ; P$ $<.001$ ). For the $\mathrm{Al}$ and PFS used for FKB327 administration, Al showed a 1.7-point lower pain score in the VAS compared with PFS $(95 \% \mathrm{Cl}$, 3.3 to $-0.1 ; P=.035)$. Gender, age, body weight, and population (healthy subject or patient) were not identified for differences in injectionsite pain intensity.

Conclusion: FKB327 showed a significant advantage in terms of injection-site pain intensity compared with the RP, as well as lack of inferiority for both Al and PFS versus RS.

Disclosure of Interests: Rieke Alten Grant/research support from: BristolMyers Squibb, Speakers bureau: Bristol-Myers Squibb, Mark C. Genovese Grant/research support from: Sanofi/Genzyme, Genentech/Roche, RPharm, Consultant for: Sanofi/Genzyme, Genentech/Roche, RPharm, Malcolm Boyce: None declared, Takuma Yonemura Grant/research support from: I have received a research grant from FKB for conducting the clinical study., Takahiro Ito Employee of: I am an employee of Fujifilm Kyowa Kirin Biologics., Herbert Kellner Grant/research support from: Roche, Consultant for: Roche

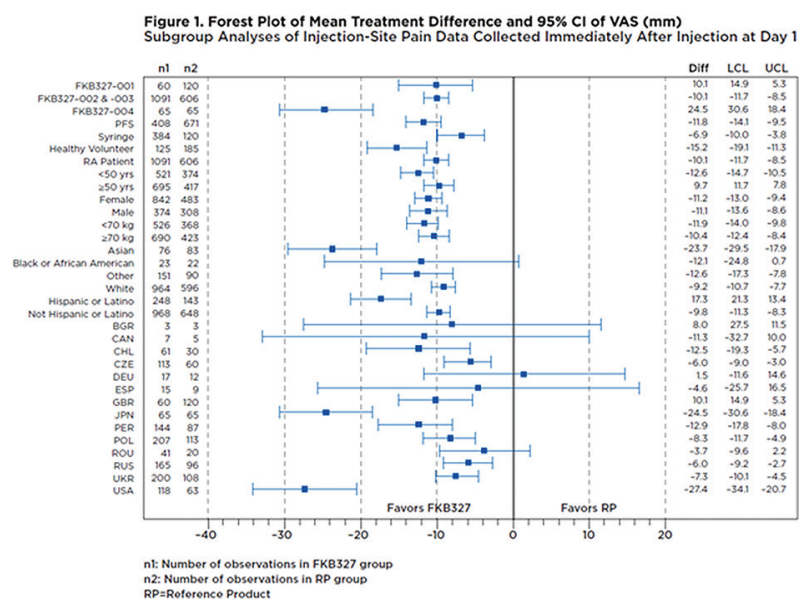

DOI: 10.1136/annrheumdis-2019-eular.1292

\section{FRI0076 THE RISK OF TUBERCULOSIS IN PATIENTS WITH IMMUNE-MEDIATED RHEUMATIC DISORDERS RECEIVING BIOLOGICAL THERAPY: A 15-YEAR EXPERIENCE IN A ROMANIAN COHORT}

ANCUTA CODRINA ${ }^{1,2}$, Cristina Pomirleanu ${ }^{1,2}$, Georgiana Strugariu $^{1}$, Luiza Petrariu ${ }^{1}$, Raluca Paiu ${ }^{1}$, Eugen Ancuta ${ }^{3}$, Codruta Bran ${ }^{4}$, Rodica Chirieac ${ }^{5}$. ${ }^{1}$ Clinical Rehabilitation Hospital, lasi, Romania; ${ }^{2}$ Grigore T Popa University of Medicine and Pharmacy, Iasi, Romania; ${ }^{3}$ Elena Doamna Clinical Hospital, lasi, Romania; ${ }^{4}$ Sfantul loan cel Nou Emergency Clinical Hospital, Suceava, Romania; ${ }^{5}$ SANOCARE Medical and Research Center, lasi, Romania

Background: The risk of tuberculosis (TB), either reactivation of latent TB or de novo infection, remains a point of interest in patients with immunemediated rheumatic pathology on biological therapy, particularly TNFo inhibitors

Objectives: We aimed to evaluate the risk and to identify predictors for $T B$ in biologics users among patients with rheumatoid arthritis (RA), 
psoriatic arthritis (PsA) and ankylosing spondylitis (AS) in North East Romania from 2003 to 2018.

Methods: We performed a hospital-based retrospective cohort study in consecutive adult patients receiving their first biologic agent (TNF or nonTNF drugs) according to local recommendations in two academic centers. Patients were classified based on the initial TB/latent TB screening test: the tuberculin skin test (positive if $>5 \mathrm{~mm}$, TST group) or interferon gamma release assays (positive if $>0.35 \mathrm{IU} / \mathrm{mL}$ QuantiFERON-TB gold, QFT group); retesting was done regularly if negative initial.

Data about drug efficacy was recorded every 24-weeks based on standard scores (DAS28-ESR for RA, DAPSA for PsA, ASDAS-CRP for AS), while TB risks at the end of the study or prior to switching were determined as hazard ratio $(\mathrm{HR})$ with $95 \%$ confidence interval $(\mathrm{Cl})$ using cox regression.

Statistical analysis was performed in SPSS-19, $p<0.05$, on subgroups of patients depending on whether positive TST or QFT at baseline.

Results: 673 patients (360 RA, 116 PsA, 195 AS) were recruited; 55 of them (33 RA, 13 AS, 9 PsA) had latent TB at baseline and received chemoprophylaxis with isoniazid before starting biologics according to local policy. Fourteen active TB were identified, the majority of them occurred within one year of biologics (ranging 6 to 52 months), as follows: three pulmonary TB in AS (two in etanercept, one adalimumab), one case of pulmonary TB in PSA (infliximab) and ten cases of RA (six pulmonary TB in abatacept, two adalimumab, etanercept and two infliximab; one ganglionar infection with infliximab; one peritoneal under certolizumab; one pulmonary and pleural under infliximab biosimilar; one pulmonary and peritoneal TB under adalimumab). The rate of active TB was 5/321.32 patient-years for infliximab, 4/594.64 for adalimumab, 1/38.39 patient-years for certolizumab, 3/721.51 for etanercept and 1/21.12 patient-years for abatacept, respectively. We reported an increased risk of TB disease in anti-TNF monoclonal antibodies users vs. soluble receptor, with an incidence ratio of $2.66(p<0.05)$. Interestingly, no significant TB risk factors were demonstrated in our cohort; baseline latent TB, previous active TB, chronic glucocorticoid use, high disease activity at baseline, comorbidities, concurrent synthetic drugs were not associated with increased risk $(p>0.05)$. In addition, we described 18 new patients with positive QFT when TB retesting as per protocol, classified as latent TB and requiring chemoprophylaxis.

Conclusion: The risk of tuberculosis remains a reality in biologics, despite extensive screening and prevention methods. The risk is variable with different drugs, higher with TNF inhibitors and relatively low in newer biologics, emphasizing a risk-stratification when selecting biologics in such patients.

Disclosure of Interests: CODRINA ANCUTA Speakers bureau: Abbvie, Pfizer, Novartis, MSD, Roche, Biogen, UCB, Lilly, Cristina Pomirleanu Speakers bureau: Abbvie, Pfizer, UCB, Georgiana Strugariu Speakers bureau: Abbvie, Pfizer, UCB, Luiza Petrariu Speakers bureau: Abbvie, Pfizer, Novartis, Raluca Paiu: None declared, Eugen Ancuta: None declared, Codruta Bran Speakers bureau: Abbvie, Pfizer, Novartis, Lilly, Roche, Rodica Chirieac: None declared

DOI: 10.1136/annrheumdis-2019-eular.3657

\section{FRI0077 SHORT DURATION OF CONVENTIONAL SYNTHETIC DISEASE-MODIFYING ANTI-RHEUMATIC DRUGS (CSDMARDS) BEFORE AND AFTER BECOMING A CSDMARD INADEQUATE RESPONDER IN RHEUMATOID ARTHRITIS PATIENTS}

Robin K Dore ${ }^{1}$, Jenya Antonova ${ }^{2}$, Huang Huan ${ }^{3}$, Magdaliz Gorritz ${ }^{3}$, Mark C. Genovese ${ }^{4} .{ }^{1}$ private practice, Tustin, CA, United States of America; ${ }^{2}$ Gilead Sciences, Foster City, CA, United States of America; ${ }^{3} I Q V I A$, Plymouth Meeting, $P A$, United States of America; ${ }^{4}$ Stanford University, Division of Immunology and Rheumatology, Stanford, CA, United States of America

Background: ACR guidelines recommend starting rheumatoid arthritis (RA) treatment with conventional-synthetic (cs)DMARD monotherapy ${ }^{1}$. If disease activity remains moderate or high, the guidelines recommend adding or switching to another csDMARD, biologic (b)DMARD, or janus kinase inhibitors (JAKi) for established RA. In early ( $<6$ months) RA, the guidelines recommend tumor necrosis factor inhibitors TNFi over JAKi and TNFi+methotrexate (MTX) over JAKi+MTX.

Objectives: This study aimed to investigate treatment patterns and duration in csDMARD IR patients.

Methods: In fully-adjudicated commercial medical and pharmacy health insurance claims database with 40 million lives annually, adult RA patients ( $\geq 2$ RA diagnoses $\geq 30$ days apart) who started csDMARD (1/1/ 2012-3/31/2017) and then switched to or added another DMARD (index date, ID) were selected. Real world treatment patterns of patients with $\geq 1$-year continuous enrollment before (baseline) and after ID (index treatment) were assessed.

Results: Among 25,104 csDMARD initiators, 10,091 (40\%) demonstrated $\mathrm{IR}$, and 7,816 met all the inclusion and exclusion criteria (median age 54 years, $26 \%$ male). The baseline csDMARD treatment lasted for a median of 4.3 months and mostly comprised of monotherapy (96\%). Upon treatment regimen change, $62 \%(n=4,869)$ initiated combination therapy $(32 \%$ csDMARD+csDMARD, $28 \%$ csDMARD+TNFi, $1.4 \%$ csDMARD+other bDMARD, $0.5 \%$ csDMARD+JAKi) and $38 \%$ initiated monotherapy $(27 \%$ on csDMARD, $10 \%$ TNFi, $0.7 \%$ other bDMARD, $0.5 \%$ JAKi). Post-switch, the median treatment duration was longer for combination therapy than monotherapy: 13.7 vs. 5.2 months $(p<0.001)$. Among next therapies, csDMARD showed the trend towards shortest durability and JAKi - toward longest durability. Among monotherapies the median treatment duration varied: 4.9 (csDMARD), 5.9 (TNFi), and 8.1 (JAKi) months ( $\mathrm{p}=0.2$, comparing all). The median duration of combination therapies lasted: 12.5 (csDMARD +CSDMARD), 14.9 (TNFi+csDMARD), and 17.2 (JAKi+csDMARD) months $(p=0.04$, comparing all).

\begin{tabular}{lcc}
\hline Characteristics & \multicolumn{2}{c}{$\begin{array}{c}\text { csDMARD IRs } \\
\text { (n=7,816) }\end{array}$} \\
\hline Index therapy allocation after csDMARD IR, (n,\%) & $\mathbf{N}$ & $\%$ \\
\hline cDMARD Monotherapy & 2,095 & 27 \\
TNFi Monotherapy & 757 & 10 \\
Other bDMARD Monotherapy & 55 & 0.7 \\
JAKi Monotherapy & 40 & 0.5 \\
cDMARD + cDMARD Combination & 2,539 & 32 \\
TNFi + cDMARD Combination & 2,178 & 28 \\
Other bDMARD + cDMARD Combination & 112 & 1.4 \\
JAKi + cDMARD Combination & 40 & 0.5 \\
Index treatment duration after csDMARD IR, months, median (IQR) & Months & $\mathbf{( I Q R )}$ \\
Monotherapy & 5.2 & 9.8 \\
csDMARD & 4.9 & 10.1 \\
TNFi & 5.9 & 10.1 \\
JAKi & 8.1 & 11.9 \\
Combination therapy & 13.7 & 15.6 \\
csDMARD+csDMARD & 12.5 & 14.8 \\
TNFi+csDMARD & 14.9 & 15.9 \\
JAKi+csDMARD & 17.2 & 11.3 \\
\hline
\end{tabular}

Conclusion: The real-world evidence suggests that treatment durability may be better for JAKi than TNFi (both monotherapy and combination). The majority of cSDMARD patients switched to another csDMARD, which showed short durability of treatment, suggesting that switching MOA may benefit these patients.

\section{REFERENCE:}

[1] Singh JA, et al. 2015 American College of Rheumatology Guideline for the Treatment of Rheumatoid Arthritis. Arthritis Rheumatol. 2016 Jan;68(1):126

Disclosure of Interests: Robin K Dore Grant/research support from: Gilead Sciences, AbbVie, Amgen, Lilly, Pfizer, Regeneron, Sanofi, Consultant for: AbbVie, Amgen, Lilly, Speakers bureau: AbbVie, Amgen, Lilly, Sanofi, Regeneron, Pfizer, UCB, Jenya Antonova Shareholder of: Gilead Sciences, Employee of: Eli Lilly and Company, Medimmune, Genentech, Gilead Sciences, Huang Huan Grant/research support from: Gilead Sciences, Magdaliz Gorritz Grant/research support from: Gilead Sciences, Mark C. Genovese Grant/research support from: Sanofi/Genzyme, Genentech/ Roche, RPharm, Consultant for: Sanofi/Genzyme, Genentech/Roche, RPharm

DOI: 10.1136/annrheumdis-2019-eular.1848

\section{FRI0078 \\ IMPACT OF THE IMPLEMENTATION OF FLUSH SERUM ON TROUGH LEVELS OF INFLIXIMAB IN PATIENTS WITH INFLAMMATORY JOINT DISEASE}

Marta Bassas Palou, Cristina Gonzalez, Ana Atares, Raimón Sanmartí, Julio Ramirez. Hospital Clinic Barcelona, Rheumatology, Barcelona, Spain

Background: The administration of intravenous biological therapies is based on the manufacturer's instructions and the administration guidelines of Spanish Medicine Agency. We observed that a considerable amount of the solution remains in the tubing of the equipment after the administration of the drug. This amount depends on the drug and the solution used for the infusion. In the particular case of Infliximab is around $7 \%$ of the total solution to infuse, so it could potentially have a relevant therapeutic impact. 